\title{
A perspective on Dyson-Schwinger equation: toy model of Pion
}

\author{
Lei Chang ${ }^{1, a}$ \\ ${ }^{1}$ CSSM, School of Chemistry and Physics University of Adelaide, Adelaide SA 5005, Australia
}

\begin{abstract}
We suggest a model representation of pion leading Bethe-Salpeter amplitude which involves a particular momentum dependence. Combining the constituent-quark propagator approximation we discuss the twist- 2 parton distribution amplitude, parton distribution function, pion elastic form factor and $\pi-\gamma$ transition form factor.
\end{abstract}

\section{Introduction}

Given its twofold roles as a conventional bound-state in quantum field theory and as the Goldstone mode associated with dynamical chiral symmetry breaking, the pion has been proven critical to explaining phenomena as diverse as the long-range nucleon-nucleon interactions. The study of the pion parton structure function and electromagnetic properties is of great interest as a fundamental test of our understanding of nonperturbative QCD.

We describe pion as bound state using the Bethe-Salpeter equation. This takes the abbreviated form:

$$
\Gamma_{\pi}(k ; P)=\int_{d q}^{\Lambda} K(q, k ; P) \chi_{\pi}(q ; P)
$$

where $\int_{d q}^{\Lambda}$ is a Poincaré-invariant regularization of the four-dimensional integral, with $\Lambda$ the ultraviolet regularization mass-scale; $q$ and $k$ are the relative momenta between the quark-antiquark pair, $P$ is the pion's four momentum and

$$
\chi_{\pi}(q ; P)=S\left(q_{+}\right) \Gamma_{\pi}(q ; P) S\left(q_{-}\right)
$$

is the pion's Poincaré-covariant Bethe-Salpeter wave-function, with $\Gamma_{\pi}$ the Bethe-Salpeter amplitude. Using isospin symmetry we label the dressed quark propagators $S\left(q_{ \pm}\right)$, where $q_{ \pm}=q \pm \frac{P}{2}$, without loss of generality. Explicitly, these take the form:

$$
S^{-1}(k)=i \gamma \cdot k A\left(k^{2}\right)+B\left(k^{2}\right)
$$

where the scalar functions $A, B$ depend on both momentum and the choice of renormalization point. With some form of quark-gluon vertex the quark-antiquark scattering kernel $K$ could be derived consistently within a symmetry preserving framework and then we can solve the quark gap equation and Bethe-Salpeter equation tegother. Some model-independent statements may also be made about the pion's Bethe-Salpeter amplitude and its relationship to the dressed quark propagator.

\footnotetext{
ae-mail: 1.chang@adelaide.edu.au
} 
In this contribution we do not try to summarize the development of Dyson-Schwinger equation apporach [1] on describing the pion properties but just rely on a simple model to cover some story of pion. We consider an algebraic model [2] where the quark propagator and the meson amplitude take of constituent mass form

$$
S^{-1}(k)=i \gamma \cdot k+M
$$

and

$$
\Gamma_{\pi}(k ; P)=i \gamma_{5} \frac{12}{5} \frac{M}{f_{\pi}} \int_{-1}^{1} d z \rho(z) \frac{M^{2}}{k^{2}+z k \cdot P+\Lambda_{\pi}^{2}},
$$

where $M$ is a dressed-quark mass, $f_{\pi}$ is the pion decay constant and we focus on the case of a massless pion, the scale in the amplitude can be set $\Lambda_{\pi}=M$ for simplify. The factor $12 / 5$ is the normalization constant needed to ensure the charge conservation. $k$ is the relative momentum of the quarks in the pion and we choose the amplitude to behave like $1 / k^{2}$ asymptotically, as this is the leading order result if one takes a one-gluon exchange interaction between the quark and antiquark. We introduce a specific Nakanishi-like representation [3] with weight function $\rho(z)$, which takes a form as

$$
\rho(z)=\frac{1}{\pi} \frac{1}{\sqrt{1-z^{2}}}
$$

In this special case we have the exact relation between $M$ and $f_{\pi}$ as $f_{\pi}=\frac{3 M}{\sqrt{10} \pi}$ and $M$ can be defined to produce the empirical $f_{\pi}$. In some more realistic cookings the mass scale in amplitude $\Lambda_{\pi}$ could be different to the constituent quark mass.

\section{Pion parton structure}

The leading twist-2 projection of the pion's Bethe-Salpeter wave function on the light-front [4] can be written as

$$
f_{\pi} \varphi_{\pi}(x)=\operatorname{tr}_{\mathrm{CD}} Z_{2} \int_{d q}^{\Lambda} \delta\left(n \cdot q_{+}-x n \cdot P\right) \gamma_{5} \gamma \cdot n \chi_{\pi}(q ; P),
$$

where: the trace is over colour and spinor indices; $Z_{2}(\zeta, \Lambda)$ is the quark wave-function renormalisation constant, with $\zeta$ the renormalisation scale; $n$ is a light-like four-vector, $n^{2}=0$.

With the algebraic model of quark propagator and Bethe-Salpeter amplitude we are able to compute the moments $\left\langle x^{m}\right\rangle=\int_{0}^{1} d x x^{m} \varphi(x)$ of the parton distribution amplitude(PDA) which yields in the form

$$
\left\langle x^{m}\right\rangle=\frac{1}{f_{\pi}(n \cdot P)^{m+1}} \operatorname{tr}_{\mathrm{CD}} Z_{2} \int_{d q}^{\Lambda}(n \cdot k)^{m} \gamma_{5} \gamma \cdot n \chi_{\pi}(q ; P)=\frac{4 \Gamma\left[\frac{3}{2}+m\right]}{\sqrt{\pi} \Gamma[3+m]},
$$

with the Euler gamma function $\Gamma[z]$. The related PDA can be read from the moments immediately as

$$
\varphi(x)=\frac{8}{\pi} \sqrt{x(1-x)}
$$

This model for the PDA follows from the precise mapping of string amplitudes in Anti-deSitter space to the light-front wavefunction of a hadron in physical space-time using holographic methods [5]. And such PDA is practically indistinguishable from the contemporary DSE prediction [4]. Whereas the scale that should be associated with the model analyses is poorly known which would be issued when it is concerned.

In Ref. [6] a corrected, leading-order expression was given for the pion's valence-quark parton distribution function(PDF). This expression produces the model-independent result that quarks dressed 
via the RL truncation carry all of the pion's light-front momentum at a characteristic hadronic scale, even if the meson amplitude is momentum dependent. We quote the form of the quark distribution function in the RL truncation here:

$$
q(x)=\operatorname{tr}_{\mathrm{CD}} \int_{d k}^{\Lambda} \delta(n \cdot k-x n \cdot P) \partial_{k}\left[\Gamma\left(k-\frac{P}{2} ;-P\right) S(k)\right] \Gamma\left(k-\frac{P}{2} ; P\right) S(k-P),
$$

The present algebraic model produce a simple form of distribution function as in the form

$$
q(x)=6 x(1-x) \propto \varphi^{2}(x)
$$

It should be emphasised that the related PDF has the power-law behavior, $1-x$, near $x=1$. That is in contrast to the QCD parton prediction.

Ezawa [7] predicted that the pion PDF would behave as $(1-x)^{2 \alpha}$ if the pion amplitude behaved as $\frac{1}{\left(k_{1}^{2}\right)^{\alpha}}$, where $k_{1}$ is the struck quark momentum. Let us take a look at asymptotic form of this model of amplitude. Introduce quarks momentum as $k_{1,2}$ with fixed pion mass $P^{2}=\left(k_{1}-k_{2}\right)^{2}$ and integrate weight function argument $z$ we can get $\Gamma_{\pi}\left(k_{1}, k_{2}\right) \propto \frac{4}{\sqrt{s^{2}-4\left(k_{1}^{2}-k_{2}^{2}\right)^{2}}}$ with $s=2\left(k_{1}^{2}+k_{2}^{2}+2 M^{2}\right)-P^{2}$. The leading oder of $k_{1}$ would be $1 / k_{1}$ if one fix $k_{2}, 1 / k_{2}^{2}$ if one fix the ratio $k_{2} / k_{1}$. Based on Ezawa's work one readily finds a PDF which behaves as $(1-x)^{2(1-0.5)}$, which is consistent with the present model calculation.

To cover QCD parton prediction one should build model that amplitude behavior like $1 / k_{2}^{2}$ asymptotically with the fixed $k_{1}$. A possible way to guarantee the asymptotic behavior $1 / k_{2}^{2}$ with fixed $k_{2}$ but taking the same value of the second central moment of PDA as the present model caluclation is to soften the weight function at boundaries $z= \pm$. A promised candidate is the two- $\delta$ function like structure of weight function. The related PDA/PDF would take the power form $1-x,(1-x)^{2}$ respectively near $x=1$ and behavior much flat in the central region of $x$. We will make a research on this possiblility throughly in future.

\section{Pion electromagnetic properties}

In this part we will take a look at the electromagnetic properties of pion in chiral limit whith the input the above model of amplitude. At leading order in the symmetry-preserving truncation scheme the pion form factor is given by

$$
P_{\mu} F\left(Q^{2}\right)=-i \operatorname{tr}_{\mathrm{CD}} \int_{d k}^{\Lambda} \Gamma_{\pi}\left(k-\frac{P}{2}+\frac{Q}{4} ;-P-\frac{Q}{2}\right) S\left(k+\frac{Q}{2}\right) \gamma_{\mu} S\left(k-\frac{Q}{2}\right) \Gamma_{\pi}\left(k-\frac{P}{2}-\frac{Q}{4} ; P-\frac{Q}{2}\right) S(k-P)
$$

where: we have repalced the dressed quark-photon vertex by its bared form due to the consistituent quark propagator approximation; $Q$ denotes the incoming photon momentum and massless pion mass shell is defined by $\left(P-\frac{Q}{2}\right)^{2}=0=\left(P+\frac{Q}{2}\right)^{2}$. Near $Q^{2}=0$ we can expand the form factor by Taylor series to order $Q^{2}$ as

$$
F\left(Q^{2}\right)=1-\frac{189}{1000} \frac{1}{f_{\pi}^{2} \pi^{2}} Q^{2}
$$

The pion charge radius is about $0.52 \mathrm{fm}^{2}$ compared to the empirical value $0.44 \mathrm{fm}^{2}$. When we take $Q^{2}$ to infinity the form factor behavior like

$$
Q^{2} F\left(Q^{2}\right) \rightarrow \frac{1}{\sqrt{Q^{2}}}
$$



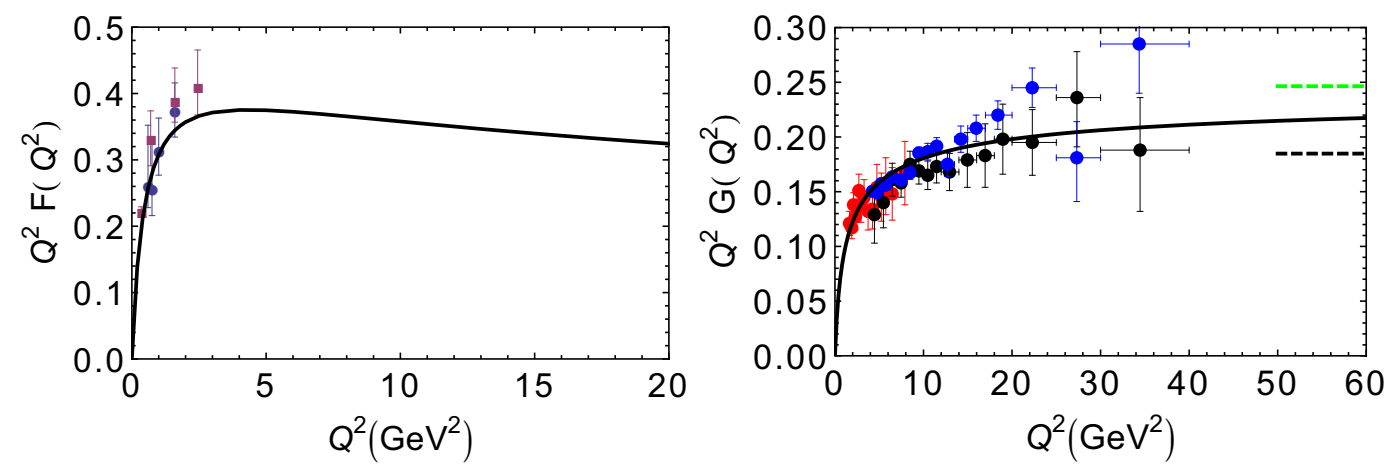

Figure 1. left panel-the momentum dependence of elastic form factor. solid line: the present model calculation and the filled circles are the data described in Ref [9]; right panel-the momentum dependence of transition form factor(divided by $4 f_{\pi} \pi^{2}$ ). solid line: the model calculation and the filled circles are the data described in Ref [10](Red for CLEO; Blue for BaBar and Black for Belle). The black dashed line denote the limit with asymptotic PDA input and green dashed line is related to the limit with the present model PDA input.

which is in contrast to the QCD prediction for pion charge form factor at suitably large $Q^{2}$. It should be noted that if one take the weight function softening at boundaries the power behavior would be $1 / Q^{2}$. It has been wildly known that large $Q^{2}$ QCD prediction would be rescured by including the pseudovector part of Bethe-Salpeter amplitude that is lacked in our model building. The model calculation of momentum dependence of $Q^{2} F\left(Q^{2}\right)$ is displayed in Fig. 1. Form factor within this simple model takes roughly same charactor as the complicate RL DS calculation [8] that there existed a maximum around $5 \mathrm{GeV}^{2}$. The results match the data well in the region $Q^{2}<2 \mathrm{GeV}^{2}$ and underestimate a little bit above. The numerical calculation could be improved by changing the value of the mass scale $M$.

For the $\pi-\gamma$ transition form factor we also suppose the bared vertex ansatz for both virtual and real photon coupling. At the leading order the triangular diagram for the transition form factor can be expressed as

$$
\frac{1}{4 \pi^{2} f_{\pi}} \epsilon_{\mu \nu \rho \sigma} k_{1}^{\rho} k_{2}^{\sigma} G\left(k_{1}^{2}\right)=-2 \operatorname{tr}_{\mathrm{D}} \int_{d k}^{\Lambda} \gamma_{\mu} S\left(k-k_{1}+k_{2}\right) \gamma_{v} \chi_{\pi}\left(k-\frac{k_{1}}{2}+\frac{k_{2}}{2} ;-k_{1}-k_{2}\right)
$$

where we set $k_{1}^{2}=Q^{2}$ the virtual photon momentum and $k_{2}^{2}=0$ the real photon momentum. Near $Q^{2}=0$ we have

$$
G\left(Q^{2}\right)=\frac{4}{5}-\frac{27}{250} \frac{1}{f_{\pi}^{2} \pi^{2}} Q^{2}
$$

It is not surprised that the present model and truncation can not produce chiral anormaly exactly because the relation between quark mass function and pion amplitude has not been preserved. A possible improvement in the model calculation can be done if we introduce the quark mass function as $M^{3} /\left(k^{2}+M^{2}\right)$ or rely on the exact consistent RL DSE calculation. We leave this topic for future.

In the large $Q^{2}$ limit we can get the analytical expression within this model as

$$
\frac{1}{4 \pi^{2} f_{\pi}} Q^{2} G\left(Q^{2} \rightarrow \infty\right) \rightarrow 8 f_{\pi} \int_{-1}^{1} d z \frac{\log \left(\frac{1+z}{2}\right)}{3(z-1)} \rho(z)=2 f_{\pi} \int_{0}^{1} d x \frac{\varphi(x)}{3(1-x)}
$$


which is consistent to the QCD predication with the input of PDA related to $\rho(z)$. The model calculation of $G$ is displayed in Fig. 1. With the increasing of momentum the form factor increases and overtakes the perturbative prediction limit $2 f_{\pi}$. At very large momentum form factor goes to the limit $\frac{4}{3} 2 f_{\pi}$ from below. At $Q^{2}=60 \mathrm{GeV}^{2}$ the dressed triangular diagram suppress form factor about $12 \%$ compared to its large $Q^{2}$ limit.

\section{Summary}

We suggest and explain a model representation of pion amplitude which is simple but provides a powerful tool to analysis the parton structure and electromagnetic properties. The corresponding relation between weight function and parton distribution amplitude is straightforward in this approach that builds a bridge to study the effect of different parton distribution amplitude on the form factors. We also like to point that he improvements, such as preserving chiral Ward-Takahashi identity, including one gluon exchange in the form factor calculation and evoluting Bethe-Salpeter amplitude with running scale, would be necessary.

\section{Acknowledgements}

I am grateful for the financial support by Prof. Anthony W. Thomas.

\section{References}

[1] I. C. Cloet,C. D. Roberts Prog. Part. Nucl. Phys. 77 (2014) 1-69.

[2] L. Chang,A. W. Thomas arXiv: 1410.8250.

[3] N. Nakanishi, Phys. Rev. 130 (1963) 1230.

[4] L. Chang, et al., Phys. Rev. Lett. 110 (2013) 132001.

[5] S. J. Brodsky, and G. F. de Teramond, Phys. Rev. Lett. 96 (2006) 201601. S. J. Brodsky, and G. F. de Teramond, Phys. Rev. D 77 (2008) 056007.

[6] L. Chang, et al., Phys. Lett. B 737 (2014) 23.

[7] Z. F. Ezawa, IL NUOVO CIMENTO 23 (1974) 271.

[8] L. Chang, et al., Phys. Rev. Lett. 111 (2013) 141802.

[9] G. Huber, et al., Phys. Rev. C. 78 (2008) 045203.

[10] J. Gronberg, et al., Phys. Rev. D 57 (1998) 33; B. Auberts, et al., Phys. Rev. D 80 (2009) 052002;

S. Uehara, et al., Phys. Rev. D 86 (2012) 092007; 